\title{
A possible hydrolysis mechanism of $\beta$-naphthyl acetate catalyzed by antibodies
}

\author{
YUAN Yu REN ${ }^{1}$, ZONG XIANG XIA ${ }^{2}$, CUI HONG \\ YANG $^{1}$, BING HUI YANG ${ }^{2}$, MING YEH ${ }^{1 *}$ \\ ${ }^{1}$ Shanghai Institute of Cell Biology, Chinese Academy \\ of Sciences, China \\ ${ }^{2}$ Shanghai Institute of Organic Chemistry, Chinese \\ Academy of Sciences, China
}

\begin{abstract}
The mechanism of ester hydrolysis has been extensively studied; however, the precise function of active-site residues in promoting catalysis is unclear. We describe here the structural models for the complex of a catalytic antibody Fv fragment with a phosphonate transition -state analogue, constructed by using gene cloning, sequencing and molecular modeling, mainly based on a known X-ray structure of a catalytic antibody. Hydrophobic and electrostatic analyses of the Fv/analog and Fv/substrate interaction suggest the hydrolysis mechanism: Tyr L91 and Tyr H97 play important roles to stabilize the $\beta$-naphthyl group of hapten through $\pi$-stack; His H35 donates a pair of free electrons at the atom NE2 to an active water and let it to be a partial hydroxide, which attacks the carbon atom of the carbonyl group of the substrate. Both His H35 and Arg L96 can form hydrogen bonds and stabilize the anionic tetrahedral intermediate formed during turnover. This mechanism emphasizes that an active water bridge may be formed during hydrolysis process.
\end{abstract}

Key words: Catalytic antibody, gene cloning, cDNA sequence, molecular modeling, ester hydrolysis mechanism.

*Correspondence to: Dr. Ming YEH, Shanghai Institute of Cell Biology, Chinese Academy of Sciences, 320 Yue-Yang Road, Shanghai 200031, China

Fax: 0086-21-64331090 E-mail: imceng@server.shcnc.ac.cn 
Hydrolysis mechanism catalyzed by antibodies

\section{INTRODUCTION}

The field of catalytic antibodies now encompasses various chemical reactions catalyzed by antibodies by using the remarkable capacity of the immune system, which generates antibodies possessing high binding affinity and structural specificity towards either the transition states or the high-energy intermediates encountered in those chemical transformations[1,2]. The creation of the "active site" is a consequence of the immunological response to the hapten structure. The majority of known catalytic antibodies have been found to catalyze the hydrolysis of esters as well as other acyl transfer reactions, which would have many potential applications in controlling biological systems. In all cases, the reaction proceeds through one or more high-energy transition states. Stable compounds that mimic the geometric and electronic characteristics of these high-energy intermediates have proved to be most useful inhibitors[3]. In particular, transition-state analogs (TSA) in which the carbonyl group has been replaced by either a charged tetrahedral phosphorus or a secondary alcohol have found wide application in the design of inhibitors to the ester hydrolysis. But, the catalytic activities exhibited by these antibodies are generally far below that of natural enzymes. It is a critical project for both biologists and chemists to improve their catalytic activities. Taking advantage of monoclonal catalytic antibodies, it is possible and important to understand the structural relationship between the TSA and the catalytic antibody raised against TSA. The most direct way to analyze the interaction between them is the X-ray crystallographic study. However, it is much difficult to get the crystals of the Fab fragment/TSA complex and few three-dimensional crystal structures are currently available[4, 5].

As reported before[6], we got two catalytic antibodies, N7 and N32, generated through immunization with a hapten 2 conjugated to a carrier protein BSA and selection of its high affinity to the short TSA 3 (Fig 1). The McAbs can hydrolyze $\beta$-naphthyl acetate 1 with very high catalytic activities (Kcat/Kuncat $=10^{6}$ ) and high specificity, something close to the natural hydrolase, porcine liver esterase, (PLE). There must be something unique lies in the structure of the antibodies. At present, we have not got the crystallographic data of the complex, but we have got the cDNA of the Fv regions of the antibodies. Two Fab fragments of antibody structures have been determined with X-ray crystallography by other groups. One is against arsonfonal, named 2F19, (Protein Data Bank, 1993 ) and the other is against a nitro-benzyl ester, named CNJ206[7, 8]. The former one has the best sequence identity with $\mathrm{N}-7$ and the latter one is a catalytic antibody. These Fabs show striking similarities in both sequences and protein structures and the overall folding of antibodies with diverse ligand specificities appears to be highly conserved with differences generally confined to the lengths and conformations of the six complementarity-determining regions (CDR loops). It seems that the Fabs of our antibodies display very similar structures, and hence their structures can be determined by computer-assisted protein modelling. In this study, we choose the 
McAb N-7, which shows more homology in DNA sequence with those of the above two reported structures, as our starting model. The coordinate of CNJ 206 is served as the main reference protein for the computer-assisted modelling of our model. The resulting model, together with comparative sequence studies, may provide insight into the mechanism.

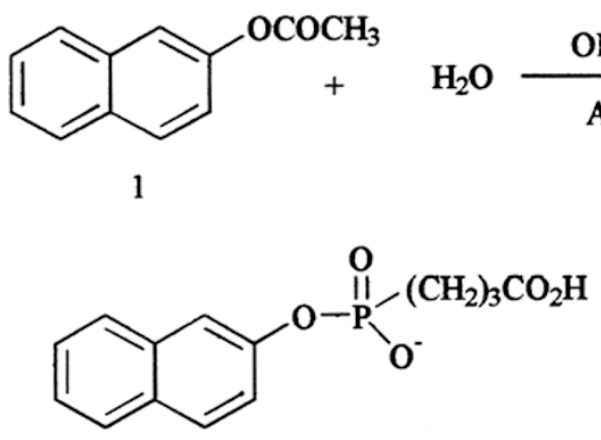

2<smiles>CP(=O)([O-])Oc1ccc2ccccc2c1</smiles>

3

Fig 1. Diagrams of the hydrolysis catalyzed by antibody N-7 and of the compounds used in this study. Compound 1 is the substrate ( $\beta$-naphthyl acetate); compounds 2 and 3 are different transition state analogs used in this study: 2 is the TSA hapten used to elicit N-7 and 3 is a short TSA used to select this catalytic antibody.

\section{MATERIALS AND METHODS}

\section{Reagents}

Klenow DNA polymerase, Moloney murine leukaemia virus (MMLV) reverse transcriptase were obtained from BRL (Grand Island, NY). Restriction enzymes (Sma I, Sac I and Xho I) were obtained either from Promega or Sino-American Biotech Co. and were used with the recommended buffer. TaqTrack sequencing Kit was from Promega. Other reagents were of molecular biology grade, pBluescript/SK vector was stored in our laboratory.

\section{Oligonucleotide primer design}

Two pairs of degenerate primers common to VH or VL were synthesized according to the universal primers for murine $\mathrm{V}$ genes designed by Orlandi et al.[9] with some modification (Tab 1).

\section{$R T-P C R$ and gene cloning}

Total RNA was extracted from N-7 hybridoma cell line cells by the guanidine isothiocyanate method. The reverse transcription reaction was performed using $10 \mu \mathrm{g}$ of the extracted total RNA as template. $4 \mu 15 \mathrm{x}$ first strand buffer $\left(250 \mathrm{mM}\right.$ Tris- $\mathrm{HCl}, \mathrm{pH} 8.0,375 \mathrm{mM} \mathrm{KCl}, 15 \mathrm{mM} \mathrm{MgCl}{ }_{2}$ ), $2 \mu \mathrm{l} 0.1 \mathrm{M} \mathrm{DTT}, 2 \mu 15 \mathrm{mM}$ dNTPs and $1 \mu \mathrm{l} \mathrm{FOR}$ primer $\left(\mathrm{J}_{\mathrm{H}} \mathrm{FOR}\right.$ or $\mathrm{J}_{\mathrm{K}}$ FOR) were added to the template to form the reaction mixture. Then, the reaction mixtures were denatured at $65{ }^{\circ} \mathrm{C}$ for $5 \mathrm{~min}$, and gradually cooled down to room temperature. One microlitre (200U) MMLV reverse transcriptase was added, followed by incubation at $37{ }^{\circ} \mathrm{C}$ for $1 \mathrm{~h}$ and denatured at $70{ }^{\circ} \mathrm{C}$ for 10 min. 1/5 of it was then taken as a template in PCR. The PCR reaction mixture consisted of $4 \mu 1$ of 
Hydrolysis mechanism catalyzed by antibodies

Tab 1. Primers for $\mathrm{V}$ region genes of heavy and light chains
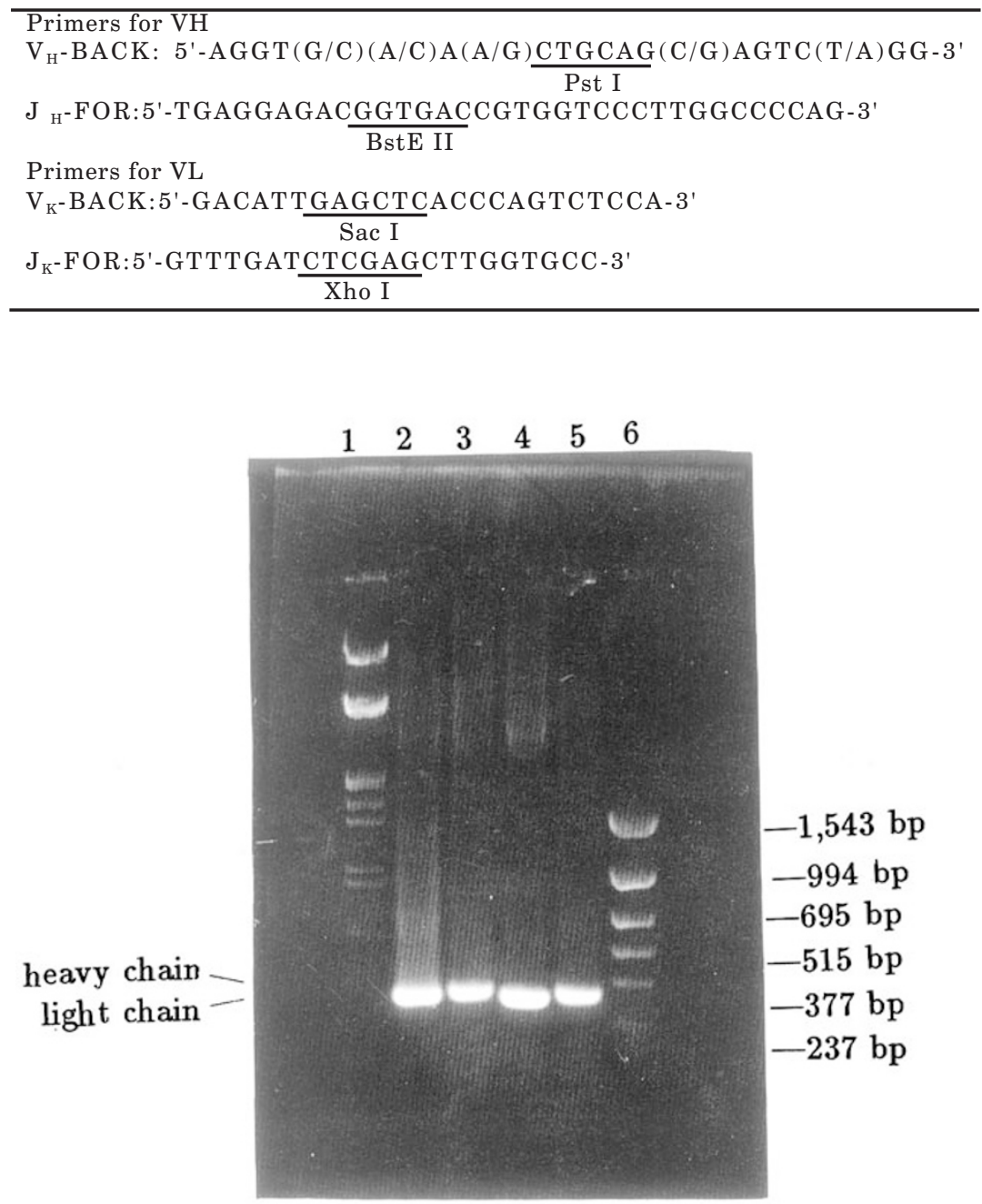

Fig 2. The PCR products of heavy chain and light chain of N-7 and N-32. (In $2 \%$ agars) Lane 1: $\lambda /$ Hind III + EcoRI PCR Markers

Lane 2: PCR product of $\mathrm{N}-32$ light chain $\left(\mathrm{V}_{\mathrm{K}} \mathrm{BACK} / \mathrm{J}_{\mathrm{K}} \mathrm{FOR}\right)$

Lane 3: PCR product of $\mathrm{N}-32$ heavy chain $\left(\mathrm{V}_{\mathrm{H}} \mathrm{BACK} / \mathrm{J}_{\mathrm{H}} \mathrm{FOR}\right)$

Lane 4: PCR product of N-7 light chain $\left(\mathrm{V}_{\mathrm{K}} \mathrm{BACK} / \mathrm{J}_{\mathrm{K}} \mathrm{FOR}\right)$

Lane 5: PCR product of $\mathrm{N}-7$ heavy chain $\left(\mathrm{V}_{\mathrm{H}} \mathrm{BACK} / \mathrm{J}_{\mathrm{H}} \mathrm{FOR}\right)$

Lane 6: PBR 322 PCR Markers (SABC, MG0781, digested by 6 endonucleases)

cDNA template, $5 \mu \mathrm{l}$ of $10 \mathrm{x}$ buffer for Taq DNA polymerase, $2 \mu \mathrm{l}$ of $5 \mathrm{mM}$ dNTPs, 25 pmol primer FOR, 25 pmol primer BACK. After denaturation of DNA mixture at $94{ }^{\circ} \mathrm{C}$ for $5 \mathrm{~min}, 1 \mu \mathrm{l}(3 \mathrm{U})$ Taq DNA polymerase was added and 35 cycles of $60 \mathrm{~s}$ at $94{ }^{\circ} \mathrm{C} / 45 \mathrm{~s}$ at $55{ }^{\circ} \mathrm{C} / 90 \mathrm{~s}$ at $72{ }^{\circ} \mathrm{C}$ were performed.

The cDNA products of light and heavy chains prepared by PCR were purified and cloned into 
the pBluescript/SK vector using standard methods[10]. Recombinant clones were selected and the presence of the inserted sequences was verified by PCR. (See Fig 2)

\section{Sequence determination}

Plasmid DNA was extracted by alkaline method, and the sequence was determined by the dideoxy (Sanger) sequencing method, using a TaqTrack Sequencing Kit. For each gene, two independent clones were analyzed until consistent sequences were obtained. The complementary determining region (CDR) and framework region (FR) in deduced amino acid sequences were defined according to Kabat et al[11].

\section{Computer modeling}

Molecular modelling was performed on a Silicon Graphics Iris Indigo 2 workstation using the commercial software packages X-plor[12] and FRODO[13]. Two high resolution X-ray crystal structures, those of $2 \mathrm{~F} 19$ and CNJ206 were used as template structure to create two independent Fv models. Each model was constructed as the following steps. First, the atomic coordinates of the structurally conserved regions (SCR) backbone and identical amino acids were copied from the template structures. Second, amino acids were replaced, inserted or deleted according to our Fv primary sequence. The $\mathrm{H} 3$ region is very short in antibody $\mathrm{N}-7$ and unlike most other antobodies, but the 5 residues in the $\mathrm{H} 3$ region is most conserved, we found that these residues can form very short $\beta$-sheet in several X-ray structures. So, we manupilated these residues as a very short $\beta$-sheet. During this computer mutagenesis, the coordinates of each side chain were oriented similarly to the corresponding side chains in the template structures. Sterical overlaps were relieved by relaxation of mutated groups by early minimization. Refinement was performed stepwise and groupwise, starting with regions containing randomly generated loops, followed by mutated residues in SCR, and ending with final minimization of all atoms.

The model deduced from CNJ206 possesses the best set of main chain conformation by Ramachandran analysis. We chose this model as the final template model. Some bad main conformation of the model was modified with the reference of the structures of 2 F 19 . The hapten or substrate was added first according to the structures of the deduced model and the known CNJ206 Fab/hapten complex structure, then using rigid-body docking algorithm to make it more compatible[14]. The final model was then generated by subjecting the derived model to further energy minimization and molecular dynamics to get more compatible interaction.

\section{RESULTS}

\section{$V$ gene sequences of the monoclonal catalytic antibody $N-7$}

The nucleotide sequences and the deduced amino acid sequences of the $\mathrm{VH}$ and VL of the catalytic antibody N-7, together with those of the reference antibodies CNJ206 and 2F19 are listed in Fig 3.

There are full of aromatic residues within the CDR regions of the McAb, N-7, it suggests that some of them may play an important role in hydrolyzing process.

\section{Interaction of the hapten with the active site}

The model suggests that the surface of Fab contains a long groove which represents a possible location for both hapten binding and catalytic activities. It is possible to dock the hapten into this model cleft with some confidence due to the shape and the electrostatic compatibility of these two structures. Using this criterion, the phosphonate of hapten was placed in the antigen-binding site above the 
Hydrolysis mechanism catalyzed by antibodies

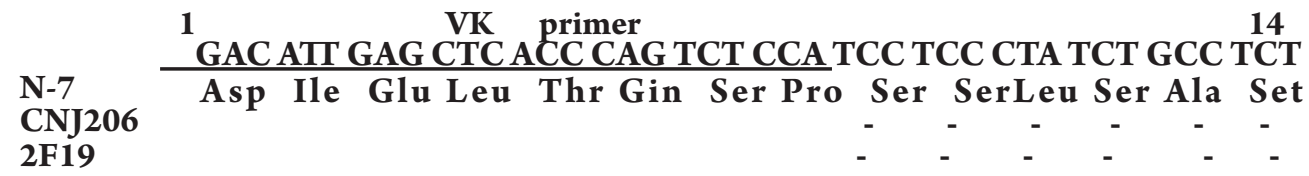

15 CTG GGA GAA AGA GTC AGT CTC ACT TGC CGG GCA AGT CAG AAT N-7 Leu Gly Glu ArgVal Set Leu Thr CysglyAla Ser Gin Asn

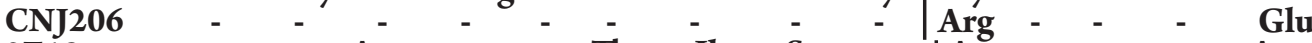
2F19 - - Asp - - Thr Ile Ser - |Arg - - - Asp

29 ATA AGT GGT TAC TTA GGC TGG CTT CAG CAG AAA CCA GAT GGA N-7 2F19 Ile Set GIy TyrLeu Gly| Trp Leu Gin GInLys Pro Asp GIy ACT TTT AAA CGC CTG ATC TAC GGC GCA TCC ATT TAA GAT TĆT Thr Phe. Lys ArgLeu Ile Tyr| Gly AlaSer Ile Leu Asp Set 2F19

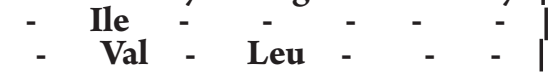
Tyr Thr - Arg - Hi 57 AT GCC CCA AAA AGG TTC AGT GC AGA AGG TCT GGG TCA GAT \begin{tabular}{llll}
- & - & - & Ser| \\
\hline & Asn & - & Asn!
\end{tabular} CDR1 Tyr His -

N-7 CNJ206 2F19 Ash Ala ProLys Arg Phe Set Gly Arg Arg SerGly Set Asp

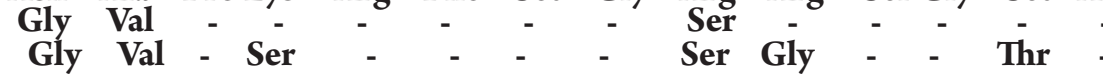
$71 \quad 84$

N-7 CNJ206 2F19 TAT TCTCTC ACC ATC AGC AGC CT GAG TCT GAA GAT TTT GCA Tyr Set Leu Thr Ile Ser Ser Ile Glu SerGlu Asp Phe Ala

CNJ206 2F19

$$
85
$$

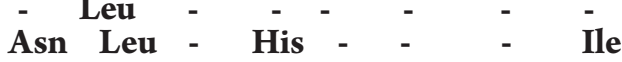
GAC TAT TAT TGT ATA CAA TAT GCT AGT TAT CCT CGG ACG TTC Asp Tyr Tyr Cys |Ile Gln Tyr Ala Ser Tyr ProArg Thr|Phe

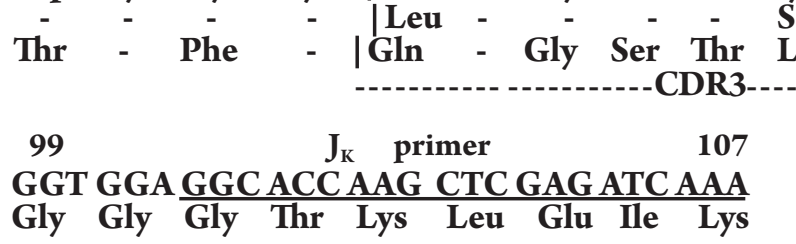

Gly Gly Gly Thr Lys Leu Glu Ile Lys

N-7 206 2F19

Fig 3a. Nucleotide and deduced amino acid sequence of N-7 K, together with the amino sequences of the VK chain of reference antibodies CNJ206 and 2F19. Dashes (-) denote sequence identity of CNJ206 or $2 \mathrm{~F} 19$ to N-7. Dots (.) denote the default sequence. 


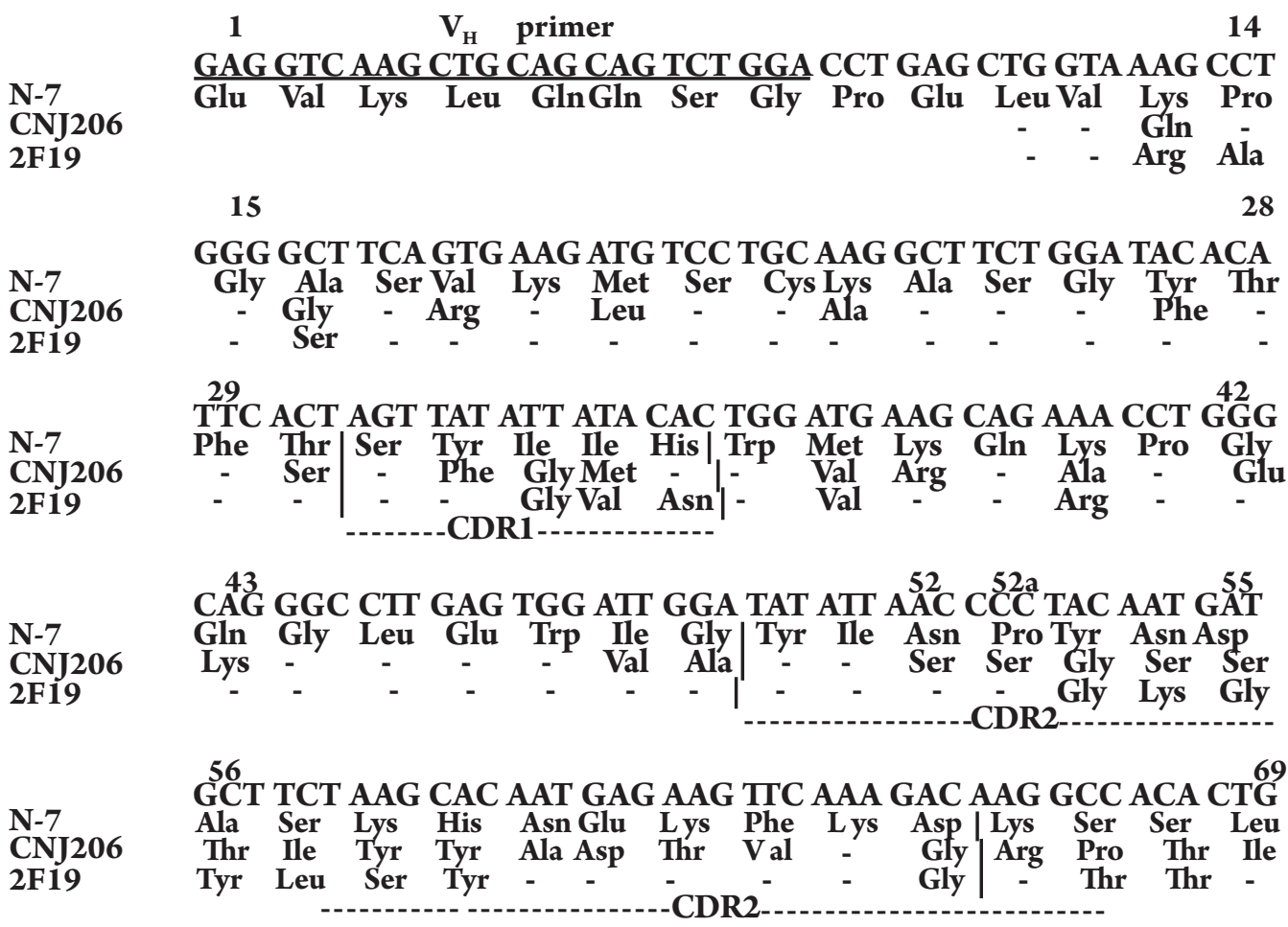

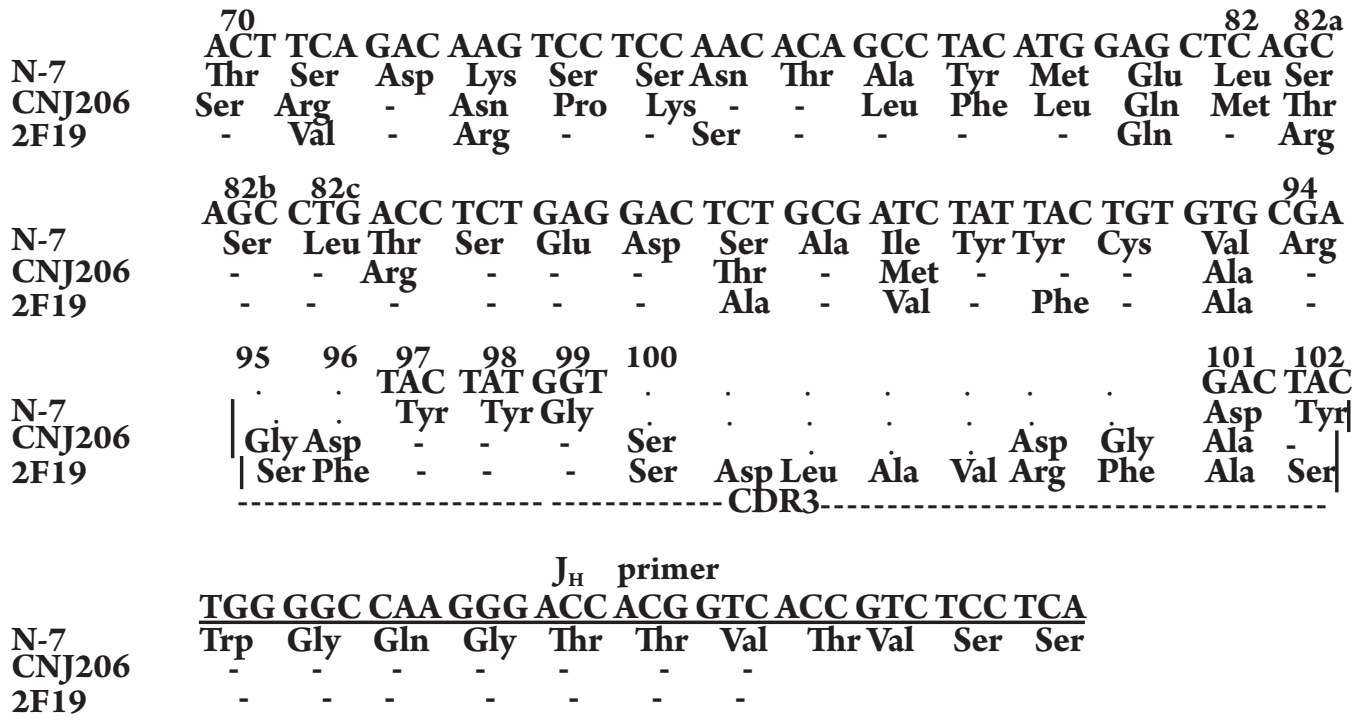

Fig 3b. Nucleotide and deduced amino acid sequence of N-7 H, together with the amino sequences of the $\mathrm{VH}$ chain of reference antibodies CNJ206 and 2 F19. Dashes (-) denote sequence identity of CNJ206 or 2 F19 to $\mathrm{N}-7$. Dots (.) denote the default sequence. 
Hydrolysis mechanism catalyzed by antibodies

side chain of arginine L96 and the docked structure was manipulated to minimize any steric clash between the aromatic side chains of the hapten and the walls of the binding pocket.

The TSA buries most of its accessible surface in the complex in a cavity mainly composed of the $\mathrm{L}$ and $\mathrm{H}$ chains of CDR3. Compound 3 is oriented so that the phosphonate is close to the outside of the antibody whereas the hydrophobic $\beta$-naphthyl group, is buried deep inside the cavity. The cavity contains a large proportion of aromatic residues and the $\beta$-naphthyl group of 3 is in a very hydrophobic environment at the bottom of the active cleft. The $\beta$-naphthyl ring of the TSA is surrounded by two tyrosine residues Tyr H97 and Tyr L91. An arginine residue, Arg L96 exposed at the bottom of the pocket represents a conserved feature of antibodies that bind phosphorus oxyanions where the side chain guanidinium group of His H35 makes both hydrogen-binding and electrostatic interactions with one of the oxygen atom of the hapten.

\section{DISCUSSION}

\section{Catalytic mechanism}

The base-catalyzed hydrolysis of esters proceeds through the formation of a negatively-charged tetrahedral transition state. Stabilization of this transition state would decrease the activity energy barrier and therefore increase the reactivity. In the dynamic analysis experiment, the hydrolysis was taken place at $\mathrm{pH}=8$, so hydroxide attack would occur without significant base catalysis, and an important effect of the antibody would be due to oxyanion stabilization. In the model there are three potential hydrogen bonds to the negatively charged phosphonate, which help to stabilize the transition-state analog. Additional contribution to catalysis in the model could come from aromatic residues and a nucleophilic attack on the carbonyl of the ester substrate by a partial ionized hydroxide induced by one of the active residues.

The mechanism of the hydrolysis of p-nitrophenyl ester has been reported[15, 16]. Several polar residues, such as arginine, tyrosine and histidine in CDRH1, CDRH3 and CDRL3, play crucial important roles in the hydrolysis mechanism. As for our model, two residues play a key role in the catalytic mechanism of hydrolysis. They are histidine H35 and arginine L96. The histidine H35, which is located approximately $6 A$ from the phosphorus atom of hapten, can form hydrogen bond with the oxygen atom of the hapten. The guanidinium group of Arg L96 can also form a hydrogen bond with another oxygen atom of the hapten and it might function to stabilize the anionic tetrahedral intermediate formed during turnover. There are two residues also play important roles to stabilize the $\beta$-naphthyl group of hapten through $\pi$-stack. They are Tyr L91 and Tyr H97. The $\beta$-naphthyl group deeply buried between the two aromatic groups of the tyrosine (within $3 \mathrm{~A}$ distance), which can stabilize the transition state further (Fig 4). Comparing our structure with the 


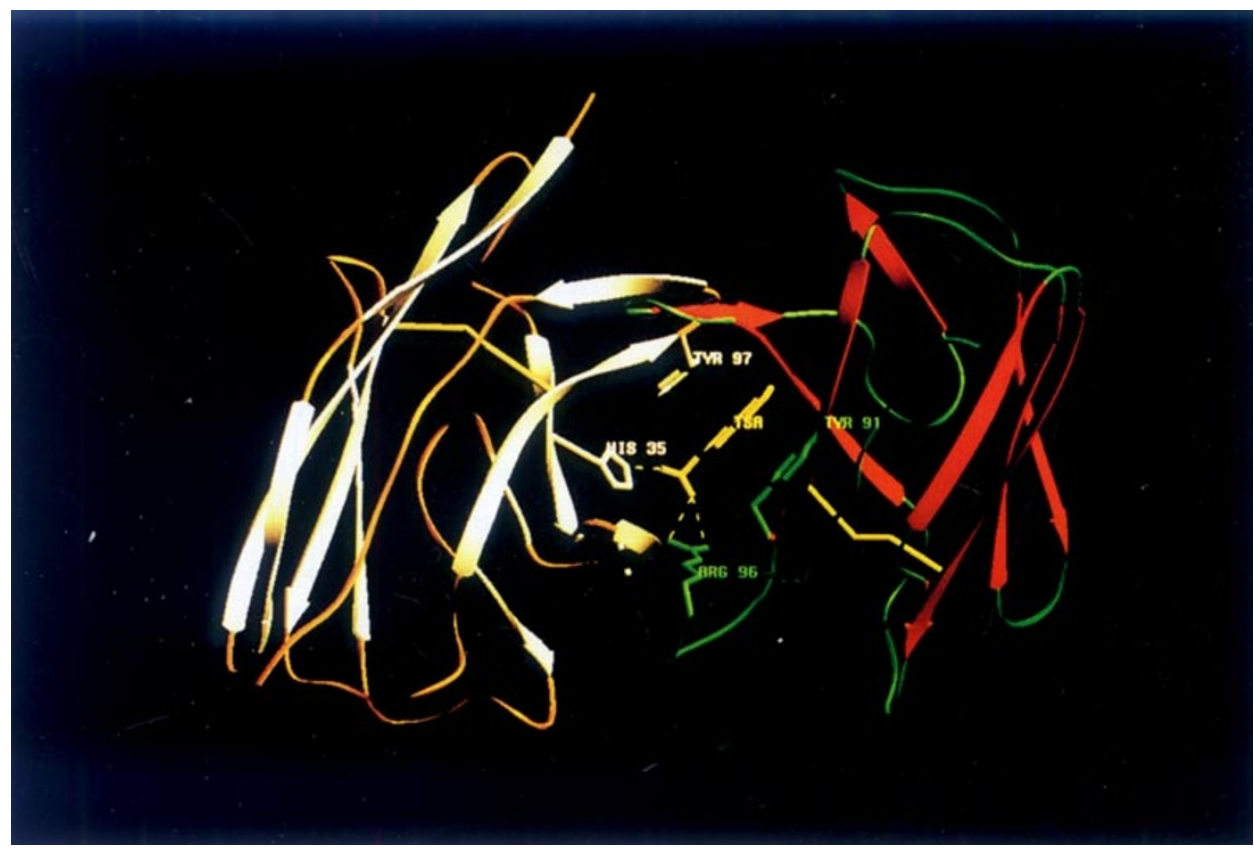

Fig 4. Hydrogen bonds in the combining site of $\mathrm{N}-7$ invoved in the stabilization of TSA 3 . The red and green ones stand for the $\beta$-sheet and the random coil of the light chain, respectively. The white and yellow ones stand for the $\beta$-sheet and random coil of the heavy chain, respectively. The hydrogen bonds are formed between the TSA and the combinding site of N-7 by two partially negatively charged oxygens of the phosphonate of theTSA with the NH1, NH2 of the side chain of Arg L96 (distance $2.87 \AA$ and 2.97 $A$ respectively) and by NE2 of His $\mathrm{H} 35$ (distance $2.97 \AA$ ). The aromatic side chains of Tyr L91 and Tyr H97 help to stabilize the $\beta$-phosphonate group of the TSA. As for the Fv/substrate complex, the NE2 of His H35 may interact with the partially positive carbon atom of the carbonyl group of the substrate through an active water bridge. The mechanism is similar to that of the hydrolysis of p-nitrophenyl ester catalyzed by antibody $43 \mathrm{C} 9[18,19]$. The figure is prepared by using the program package SETOR[20].

antibody 48G7, which catalyzes the hydrolysis of p-nitrophenyl alkyl ester[17], we found several active-site residues are the same, such as Arg L96 and His H35. Structural and kinetic studies indicate that there is no participation of a protein nucleophile in the hydrolysis of p-nitrophenyl ester. Several active-site residues may directly stabilize the tetrahedral intermediate. Mutagenesis, chemical modification studies, and structural data suggest that His H35 and Arg L96 are important to catalyze the hydrolysis of p-nitrophenyl ester. Mutation of His H35 to Glu H35 reduces Kcat by a factor of 30 and mutation of Arg L96 to Gln L96 only leads to modest reductions in Kcat[17]. So the roles of His H35 and Arg L96 are different during ester hydrolysis. It was also reported that water attacking was essential during the 
Hydrolysis mechanism catalyzed by antibodies

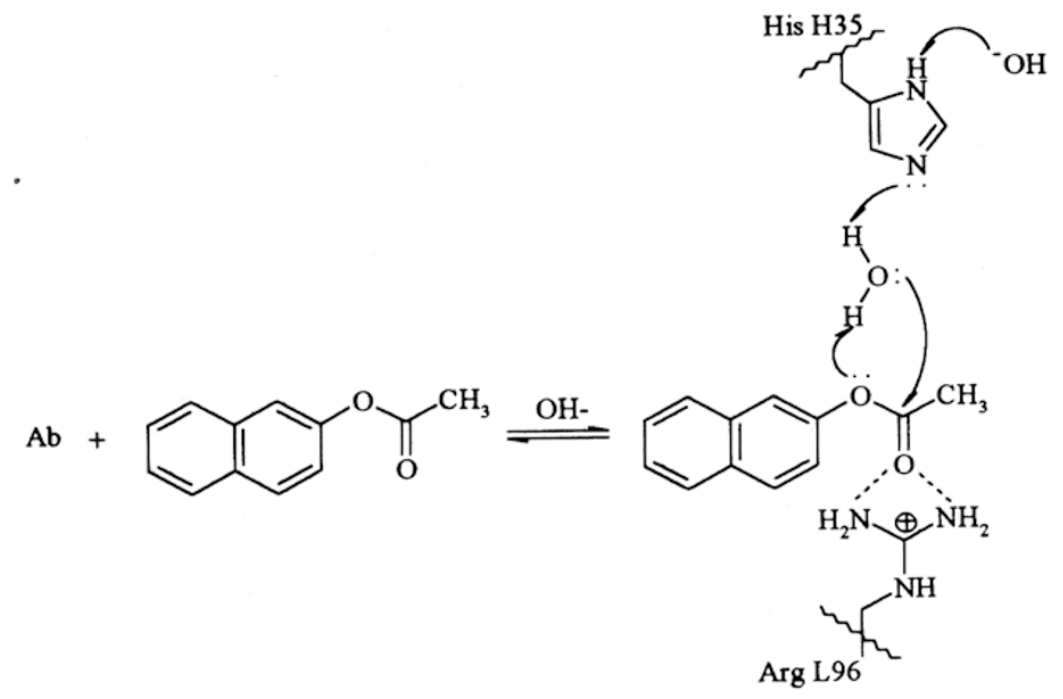

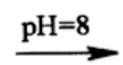<smiles>Oc1ccc2ccccc2c1</smiles><smiles>CC(=O)[O-]</smiles><smiles>CCCCC(C)(C)NC(N)N</smiles>

Fig 5. Hydrolysis mechanism of $\beta$-naphthyl ester catalyzed by antibody N-7. At the first step, His H35 donates a pair of free electrons at the atom NE2 to an active water and let it to be a partial hydroxide, which attacks the carbon atom of the carbonyl group of the substrate. Then, together with Arg L96, stabilizes the newly-formed oxyanion intermediate by electrostatic and hydrogen-bonding interactions. It seems to form in a reversable step at this time. Hydroxide ion is then believed to attack the acylantibody to form the antibody-product complex from which the ligands dissociate to complete the catalytic cycle. It is a unreversable step which favors the product.

catalytic pathway[5]. So we suggest that H35 plays a crucial role in the catalysis of $\beta$-naphthyl acetate: First, it donates a pair of free electrons at the atom NE2 
to an active water and let it to be a partial hydroxide, which attacks the carbon atom of the carbonyl group in the substrate. Then, together with Arg L96, it stabilizes the newly-formed oxyanion intermediate. The suggested hydrolysis mechanism catalyzed by antibody N-7 can be seen from Fig 5 .

It has been noted that catalytic antibodies often fail to process multiple substrate per active site in an effective way, an important feature of any true catalyst and a hallmark of enzymes. One possible cause of this lack of processing may be product inhibition. Since transition state analog haptens and the reaction product will invariably contain identical groups, product inhibition can be expected and is often observed in antibody-catalyzed reactions. But, indeed, the delocalized negative charge of the product (ionized $\beta$-naphthylate) at $\mathrm{pH}=8$ is expected to disfavor binding in the hydrophobic cavity found in the Fv structure. In our model, the CDRH3 is very short, it contains only 5 residues. Taking advantage of the short CDRH3, the antibody N-7 forms an active site with a long-groove shape, which appears to be more suitable for product release than a deep specific pocket.

\section{ACKNOWLEDGEMEMTS}

The authors want to express their sincere thanks to Dr. Marcel Knossow at CNRS for the crystal coordination of CNJ206. The study is supported by the Director's Grant of Shanghai Institute of Cell Biology and World Laboratory.

\section{REFERENCES}

[1] Lerner RA, Benkovic SJ, Schultz PG. At the crossroads of chemistry and immunology: Catalytic antibodies. Science 1991; 252:659-67.

[2] Benkovic SJ. Catalytic antibodies. Ann Rev Biochem 1992; 61:29-54.

[3] Wolfenden R. Analog approaches to the structure of the transition state in enzyme reactions. Acc Chem Res 1972; 5:10-8.

[4] Charbonnier JB, Carpenter E, Gigant B et al. Crystal structure of the complex of a catalytic antibody Fab fragment with a transition state analog: Structural similarities in esterase-like catalytic antibodies. Proc Natl Acad Sci USA 1995; 92:11721-5.

[5] Gigant B, Charbonnier JB, Eshhar Z et al. X-ray structures of a hydrolytic antibody and of complexes elucidate catalytic pathway from substrate binding and transition state stabilization through water attack and product release. Proc Natl Acad Sci USA 1997; 94:7857-61.

[6] Yuan YR, Yang BH, Ji YY. An improved rapid method for selecting monoclonal antibodies with high catalytic activities. J Prot Chem 1997; 8:733-8.

[7] Zemel R, Schindler DG, Tawfik DS et al. Differences in the biochemical properties of esterolytic antibodies. Molec Immun 1994; 31:127-37.

[8] Golinelli-Pimpaneau B, Gigant B, Bizebard T et al. Crystal structure of a catalytic antibody Fab with esterase-like activity. Structure 1994; 2:175-83.

[9] Orlandi R, Gussow DH, Jones DT et al. Cloning immunoglobulin variable domain for expression by the polymerase chain reaction. Proc Natl Acad Sci USA 1989; 86:3833-7.

[10] Sambrook J, Eritsch EF, Maniatis T. (1989) Molecular Cloning: a laboratory manual. 2nd edn. Cold Spring Harbor Laboratory. Cold Harbor, NY.

[11] Kabat EA, Wu TT, Perry HM et al. (1991) Sequences of Proteins of Immunological Interest Vol.1 5th edn. US Department of Health and Human Services, MD. 


\section{Hydrolysis mechanism catalyzed by antibodies}

[12] Brunger AT, Krukowski A, Erickson JW. Slow-cooling protocols for crystallographic refinement by simulated annealing. Acta Crystalogr. A 1990; 46:585-93.

[13] Cambillau C, Horjales E. Tom: A FRODO subpackage for protein ligand fitting with interactive energy minimization. J Mol Graphics 1987; 5:174-7.

[14] Cherfils J, Janin J. Simulating molecular recognition. Curr Opin Struct Biol 1993; 3:265-9.

[15] Gigant B, Charbonnier JB, Golinelli-Pimpaneau B et al. Mechanism of inactivation of a catalytic antobody by $\pi$-nitrophenyl esters. Eur J Biochem 1997; 246:471-6.

[16] Wedemayer GJ, Wang LH, Patten PA et al. Crystal structures of the free and liganded form of an esterolytic catalytic antibody. J Mol Biol 1997; 268:390-400.

[17] Patten PA, Gray NS, Yang PL et al. The immunological evolution of catalysis. Science 1996; 271:1086-91.

[18] Roberts VA, Stewart JD, Benkovic SJ et al. Catalytic antibody model and mutagenesis implicate arginine in transition-state stabilization. J Mol Biol 1994; 235:1098-116.

[19] Stewart JD, Roberts VA, Thomas NR et al. Site directed mutagenesis of a catalytic antibody: an arginine and a histidine residues play key roles. Biochemistry 1994; 33:1994-2003.

[20] Evans SV. SETOR: Hardware lighted three dimensional solved model representations of macromolecules. J Mol Graphics 1993; 11:134-8.

Received May-21-1998. Revised July-21-1998. Accepted July-23-1998. 